\title{
Notas Linguísticas sobre el Verbo en el Dialecto "Bue" de los Murui
}

HORACIO CALLE 
Aunque mi campo de especialización no es la lingüística, mi intento como antropólogo de tener una comprensión de la cultura Murui-Muinane, y de aplicar este conocimiento a la solución de su problemática socio-cultural, me llevó irremediablemente a estudiar y aprender el dialecto "Bue" de los Murui.

$\mathrm{Y}$ digo que irremediablemente porque es del todo imposible realizar un estudio serio de una cultura aborigen sin hablar fluentemente su idioma. Esto por dos razones principales. En primer lugar, en el mundo cultural del indígena existen muchos conceptos referentes a sus estructuras económica, social e ideológica que no tienen contrapartida en el mundo cultural del "blanco" y por lo tanto son casi intraducibles. Esto es cierto especialmente en lo referenre a los conceptos de la estructura ideológica, cuyo estudio es el tema favorito de muchos antropólogos hoy en día. La complejidad conceptual de estos sistemas hace más necesario aún el requisito del aprendizaje del idioma nativo. Cualquier intento de trabajar a través de intérpretes solo conducirá a superficiales aproximaciones al mundo conceptual del indígena 0 a burdas tergiversaciones del mismo. En segundo lugar, y debido a la situación de "contacto" en que viven los grupos indígenas de Colombia, con una situación caracterizada por relaciones de opresor (el "blanco") a oprimido (el indígena), de explotador a explotado, mal puede llegarse a conocer el punto de vista del indígena utilizando el lenguaje del explotador.

En otras palabras, al intentar ponerme dentro de la cultura de los Murui-Muinane, me encontré con la imperiosa necesidad de hablar en su idioma a pesar de que casi todos ellos hablan el español. Como dice Fanon (1965:42) "Hablar. Esto significa emplear una cierta sintaxis, poseer la morfología de esta o aquella lengua, pero, fundamentalmente, es asumir una cultura, soportar el peso de una civilización" (mi subrayado).

Los Murui-Muinane (mal llamados Uitotos) han habitado tradicionalmente los ríos Caraparaná e Igaraparaná (Uyocue y Cotue respectivamente en su idioma), afluentes norte del rio Putumayo, en la parte occidental de la intendencia del Amazonas, Colombia. Debido a la persecución de que fueron víctimas durante la época de las caucherías, ellos se desplegaron por toda la región y hoy se les encuentra desde el piedemonte andino (Florencia) hasta Leticia, y desde el río Caquetá (Colombia) hasta el río Napo (Perú). 
Los Murui-Muinane hablan varios dialectos intimamente ligados entre sí, tales como

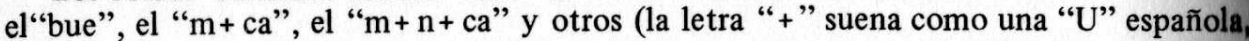
suave y un poco nasalizada. Los nombres de los dialectos son los mismos con los cuales ellos denominan a éstos). El dialecto "bue" es uno de los más extendidos.

Las anotaciones linguísticas que siguen hacen referencia al verbo entre los parlantes del "bue", y algunos aspectos de su empleo. Las presentamos sin mayor elaboración lingüística, con el solo ánimo de ofrecer "materia prima" al lingüista profesional.

\section{EL INFINITIVO}

En "bue" el infinitivo siempre tiene dos terminaciones, es decir, todos los verbos terminan en "te" o en "de":

$\begin{array}{llll}\text { Ite ser o estar } & \text { Atúde } & \text { traer } \\ \text { Rúte comer carne } & \text { Cacáde } & \text { escuchar } \\ \text { Bíte venir } & \text { Jáide } & \text { ir } \\ \text { Róte cantar } & \text { Uáide } & \text { caer } \\ \text { Jufánotejugar } & \text { Nóide } & \text { bañar } \\ \text { Yíte chupar } & \text { Jitáide } & \text { desear } \\ \text { Gúite comer } & \text { F+inóde } & \text { fabricar } \\ \text { Isíruite amar } & \text { Jenóde } & \text { buscar } \\ \text { Dorítate disparar } & \text { Facáde } & \text { ensayar } \\ \text { Dúte comer coca } & \text { Fecáde } & \text { repartir }\end{array}$

La conjugación del verbo se hace posponiendo el pronombre al infinitivo, cuya "๑" final se transforma en una "+". Como el uso del pronombre es bastante complejo en ol "bue" debido a su riqueza en pronombres, aquí solo utilizaré los pronombres "yo" y "tu", "cue" y "o" respectivamente Algunos ejemplos bastarán para ilustrar lo dicho anteriormente:

$$
\begin{aligned}
& \text { It }+ \text { cue = yo soy o estoy } \\
& \text { It }+o=\text { tú eres o estás } \\
& \text { Bit }+ \text { cue = yo vengo } \\
& \text { Bit }+o=\text { vienes } \\
& \text { Jufanot }+ \text { cue = juego } \\
& \text { Jufanot }+o=\text { juegas } \\
& \text { Ona isiruit }+ \text { cue = yo te amo } \\
& \text { Air }+ \text { j+guit }+ \text { cue = como casabe } \\
& \text { Cacad }+o \text { ? = escuchas? } \\
& \text { Jibie at }+d+\text { cue = traigo coca. }
\end{aligned}
$$

\section{LOS TIEMPOS}

Es contraproducente intentar forzar sobre el "bue" las mismas categorías de temporalidad que nosotros tenemos en español: copretérito, antepresente, pospretórito etc. Los Murui tienen sus propias formas de expresar la temporalidad, y de estas hemo: logrado clarificar las siguientes:
El Presente En el "bue" el presente puede indicarse en dos formas diferentes:

Una forma sencilla, que también se utiliza para expresar un pasado, y que se obtiene con la sola conjugación del infinitivo tal como se vió en los ejemplos a respecto (página 2), de modo que expresiones tales como "jaid+cue" bien pueden significar "me voy" o "me fui" según el contexto de la frase en que se incluyen.

Existe además una forma compuesta del presente que indica una acción que se está haciendo al instante de hablar, y que implica alguna duración, como si se estuviera haciendo por un rato prolongado:

$$
\begin{aligned}
& \text { Jufano jufanot }+ \text { cue }=\text { estoy jugando } \\
& \text { Rua faca facad }+ \text { cue }=\text { estoy ensayando canciones (rua) } \\
& \text { Ca+ gmare ro rod }+ \text { cue }=\text { esoy cantando contento }(c a+\text { gmare }) \\
& \text { Air }+\mathrm{j}+\text { gui guit }+ \text { cue }=\text { estoy comiendo casabe }(a i r+j+)
\end{aligned}
$$

El Futuro. El tiempo futuro se puede expresar en tres formas diferentes. Una de ellas es utilizando el verbo auxiliar "ir a" pero como se trata de una forma "ayudada" no la incluímos aquí en de talle. Solo daré unos ejemplos al respecto:

$$
\begin{aligned}
& \text { Jibie uaid }+ \text { cue }=\text { voy a sacar coca }(\text { jibie }) \\
& \text { Air }+j+\text { guisaid }+ \text { cue = voy a comer casabe }(\operatorname{air}+j+)
\end{aligned}
$$

Las otras dos formas si son propias del futuro, es decir las forma la declinación o conjugación del propio verbo sin necesidad de verbos auxiliares. Así:

1) Todos los verbos cuyo infinitivo termina en "de" para formar el futuro cambia la "D" por "T" y anteponen a ésta una "I" con excepción de los infinitivos cuya raiz ya termina en "I" o en "a+g".

Ejemplos:

$\begin{array}{llll}\text { F }+ \text { inod }+ \text { cue } & \text { fabrico o hago } & \text { F+inót +cue } & \text { fabricaré } \\ \text { Facad }+ \text { cue } & \text { ensayo } & \text { Facáit }+ \text { cue } & \text { ensayaré } \\ \text { Jaid }+ \text { cue } & \text { me voy } & \text { Jáit }+ \text { cue } & \text { me iré } \\ \text { At }+d+\text { cue } & \text { traigo } & \text { At }+ \text { it }+ \text { cue } & \text { traeré }\end{array}$

2) Todos los verbos cuyo infinitivo termina en "te" conservan la " $t$ ", y observan la misma regla con respecto a la intercalación de una " $i$ ", pero al formar el futuro trasladan el acento de la terminación verbal (presente), a la raíz del verbo (futuro):

$\begin{array}{llll}\text { Rot }+ \text { cue } & \text { yo canto } & \text { Róit +cue } & \text { cantaré } \\ \text { Jibie dut }+ \text { cue } & \text { como coca } & \text { Jibie dúit }+ \text { cue } & \text { comeré coca } \\ \text { Jufáno }+ \text { cue } & \text { yo juego } & \text { Jufánóit }+ \text { cue } & \text { yo jugaré }\end{array}$

La segunda forma de este futuro propio se obtiene remplazando la terminación del infinitivo, bien sea en "de" o en "te" por la particula "ye". Así:

$\begin{array}{llll}\text { Jáide } & \text { ir } & \text { Cue jáiye } & \text { yo me iré } \\ \text { Dúte } & \text { comer coca } & \text { Cue dúye } & \text { comeré coca }\end{array}$


Guáte pilar

cantar

Cue gúáye

yo pilaré Cue róye

yo cantaré

Esta forma del futuro es utilizada para expresar un futuro inmediato, algo que va suceder inmediatamente después de que se habla.

El Pasado. Igual que el futuro, el pasado puede expresarse de varias formas, y realidad no tenemos aun muy claro los criterios en los cuales se basan los parlantes "bue" para seleccionar una forma de entre las otras.

1) Como ya se indicó anteriormente el pasado y el presente se pueden indicar do igual forma, todo depende del contexto de la frase en que se encuentra. Veamos po: ejemplo con la expresión "jaid+cue" (yo me voy o yo me fui):

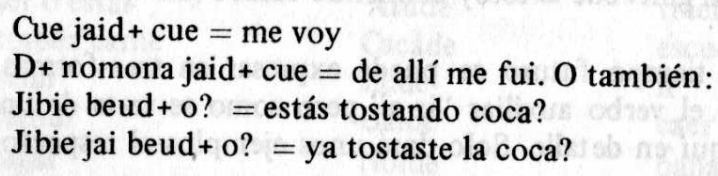

2) Existe una segunda forma de formar el pasado, la cual es muy compleja debide a la variabilidad de sus terminaciones, pero todas ellas obedecen a reglas bien definide. así:

A) Los verbos cuya raíz del infinitivo termina en "i", añaden al particula "ya",

$\begin{array}{llll}\text { Ride } & \text { llegar } & \text { Cue riya meno } & \text { después de que llog } \\ \text { Guite } & \text { comer } & \text { Cue guiya meino } & \text { después que comi } \\ \text { Bite } & \text { venir } & \text { Cue biya meino } & \text { después que vine }\end{array}$

B) Los verbos cuya raiz del infinitivo termina en "o", suprimen la "o" final agregan la partícula "ua".

$\begin{array}{llll}\text { Rote } & \text { cantar } & \text { Cue rua meino } & \text { después de que yo cant } \\ \text { C+ode } & \text { ver } & \text { Cue c+a } & \text { yo vi } \\ \text { Jocode } & \text { lavar } & \text { Cue jocua meino } & \text { después de que yo lavd } \\ \text { Jirode } & \text { beber } & \text { Cue jirua meino } & \text { después de que yo bobf }\end{array}$
C) Los verbos cuyo infinitivo tiene una raiz terminada en " $e$ ", suprimen la " $e$ "
añaden la partícula "ia":

Dobede triturar yuca $\quad 0$ bobia meino

después que ud. machucó la yuce

D) Los verbos cuya raíz del infinitivo termina en "a", añaden la partícula "ja", Asi:

$\begin{array}{llll}\begin{array}{l}\text { Macade } \\ \text { Guate }\end{array} & \begin{array}{l}\text { caminar } \\ \text { pilar }\end{array} & \begin{array}{l}\text { Cue macaja meino } \\ \text { Jibie cue guaja meino }\end{array} & \begin{array}{l}\text { después que camind } \\ \text { después que de yo } \\ \text { pilé la coca }\end{array} \\ 368 & & \end{array}$

E) Los verbos cuya raiz del infinitivo termina en " $u$ " $o$ en " $+g$ ", simplemente añaden una "a". Asi:
$\widetilde{N} a+$ gte
hablar
Cue ña + ga meino
después de que yo hablé

3) Existe una tercera posibilidad de formar el pasado, la cual se utiliza para pasados bastante lejanos en el tiempo, y se obtiene añadiendo a la raíz del infinitivo la particula "ga". Así:

$\begin{array}{llll}\begin{array}{l}\text { Doritate } \\ \text { Yote }\end{array} & \begin{array}{l}\text { disparar } \\ \text { decir }\end{array} & \text { Cue doritaga } & \text { yo disparé } \\ \text { O yoga } & \text { ud. dijo }\end{array}$

Como lo anoté anteriormente aun no tenemos lo suficientemente claro cuales son los criterios que los parlantes de "bue" tienen en cuenta para seleccionar entre las tres formas de hacer el pasado.

El Imperativo. Los verbos cuyo infinitivo termina en "de" forman el imperativo simplemente suprimiendo esta terminación:

$\begin{array}{llll}\text { Jiróde } & \text { beber } & \text { Jiro } & \text { beba } \\ \text { Fatade } & \text { golpear } & \text { Fata } & \text { golpee } \\ +n+d e & \text { dormir } & +n+ & \text { duerma } \\ \text { Iside } & \text { cernir } & \text { Isi } & \text { cierna }\end{array}$

Los verbos cuyo infinitivo termina en "te" suprimen esta terminación y añaden la terminación "no", teniendo en cuenta de que la "n" se transforma en "ñ" después de "i". Así:

$\begin{array}{llll}\text { Nite } & \text { tejer } & \text { Niño } & \text { teja } \\ \text { Rote } & \text { cantar } & \text { Rono } & \text { cante } \\ \text { Facuite } & \text { levantarse } & \text { Facuiño } & \text { levántese } \\ \text { Ñatgte } & \text { hablar } & \text { Natgno } & \text { hable } \\ \text { Guite } & \text { comer } & \text { Guiño } & \text { coma }\end{array}$

\section{OTROS ASPECTOS DEL VERBO}

Existen en el dialecto "bue" una serie de expresiones que modifican al verbo en forma diferente a como lo hacemos en español. Su número es bastante grande, y pensamos presentar un informe por separado al respecto, pero como quiera que ellas están relacionadas con el verbo y sus formas, hemos considerado conveniente incluír algunas de ellas en este informe a modo de ilustración.

El énfasis. En el dialecto "bue" el énfasis puede expresarse agregándole la partícula "sa" a la palabra que se quiere enfatizar, inclusive al verbo. Esta terminación "sa" es de uso muy corriente. Veamos algunos ejemplos:

$$
\begin{array}{llll}
\text { Jaid }+ \text { cue } & \text { me voy } & \text { Jaid + cuesa } & \text { me voy, si! } \\
\text { + care bit +o } & \text { venga mañana } & \text { + care bit + osa } & \text { venga mañana, segurg } 5 !
\end{array}
$$


El deseo. En el dialecto "bue" existen claro está varios verbos que indican la actitud de desear algo, de querer algo, tales como "jitaide", o "isiruite". Pero hay una forme muy común que no es un verbo en sí, sino que debe ser utilizada con otros verbos indicando que se desea esa acción:

$\begin{array}{llll}\text { Jaid }+ \text { cue } & \text { yo voy } & \text { Cue jai iacad }+ \text { cue } & \text { yo quiero ir } \\ \text { Bit }+ \text { cue } & \text { vengo } & \text { Cue bi iacad }+ \text { cue } & \text { yo quiero venir } \\ \text { Guit }+ \text { cue } & \text { como } & \text { Cue gui iacad }+ \text { cue } & \text { yo quiero comer } \\ +\mathrm{n}+\text { de }+ \text { cue } & \text { duermo } & \text { Cue }+n+\text { iacad }+ \text { cue } & \text { yo quiero dormir } \\ \text { J+ nui jiró }+ \text { cue } & \text { tomo agua } & \text { J + nui jiró iacad }+ \text { cue quiero tomar agua } \\ \text { Ogodo yit +cue } & \text { estoy comiendo } & \text { Ogodo yi acad }+ \text { cue quiero comer banano } \\ \text { banano. } & & \end{array}$

\section{EL SINCRETISMO LINGUISTICO}

Estando como están los Murui-Muinane en una situación de contacto con I cultura colombiana, es obvio suponer que se presentan casos de sincretismo cultural, decir que los Murui-Muinane toman prestados elementos culturales de la cultura del "blanco" y los transforman de acuerdo a sus propias pautas. El lenguaje no es un excepción. Los parlantes del "bue" han tomado una serie de expresiones del español y las han adaptado a su idioma. Generalmente claro está, se adoptan aquellas palabra: que denotan conceptos nuevos para la cultura Murui, y que por lo tanto no tienon equivalentes en su idioma.

Tenemos por ejemplo el caso del tabaco. Entre los Murui-Muinane el tabaco os algo muy sagrado e importante y se le consume en varias formas como pasta o come líquido para chupar. En el idioma "bue" se le conoce con el nombre de "yéra".Pero in práctica de fumar el tabaco la aprendieron del blanco, y con la práctica el verbo "fumar" el cual ellos conjugan a la manera "bue". Así:

$$
\begin{array}{ll}
\text { Cue fumád +cue } & \text { yo fumo } \\
\text { Cue fumá iacad + cue } & \text { yo quiero fumar } \\
\text { Cue fumáñed }+ \text { cue } & \text { yo no fumo }
\end{array}
$$

E igual sucede con todos los demás verbos que ellos deseen tomar del español

Al comienzo de este artículo expresamos nuestro deseo de que las notas aquf incluídas pudieran servir de "materia prima" al lingüista profesional como materiel comparativo. Pero también dejamos claro nuestra opinión de que el conocimiento dol lenguaje es absolutamente indispensable para tener una comprensión cabal, objetiva, científica.

Sin este conocimiento del lenguaje solo puede llegarse a vulgares tergiversaciones dol mundo cultural que se desea estudiar. Tal es el caso que pasamos a narrar " continuación: Según el Dr. Ney Guzmán Gómez (1968:26) quien publicó un estudio "médico antropológico" de los indígenas de la Amazonia colombiana: "Hablan huitoto, dialecto de léxico reducido, pobre y de muchas contracciones que hacen difícil su aprendizaje. No tienen palabras para manifestar ideas abstractas como justicia verdad, virtud, castidad, ni siquiera tiempo, ni edad; esto explica su conformismo aterrador. Carecen de vocablos para manifestar afectividad (continúa el tal doctor Ney Guzmán) amor, cariño, gracias, etc. por lo cual nunca, hasta ahora con la 370 universalización del castellano, pudieron gozar del deleite de estar enamorados. No tienen en sus dialectos los verbos ser y haber, ni infinitivo. Sus sonidos son guturales y entre dientes". Y otras imbecilidades por el estilo. La ignorancia del mundo cultural de los Murui-Muinane (mal llamados Uitotos) llevó al doctorcito a hacer una serie de afirmaciones irresponsables y falsas que rayan en el racismo vulgar y en la explotación.

En léxico de los Murui-Muinane no es reducido. Es tan variado, rico y complejo como el mundo natural que les rodea, la selva tropical lluviosa, teniendo un vocablo para toda especie animal y vegetal, o fenómeno natural cualquiera observable a simple vista, sin hablar del léxico referente a sus relaciones sociales y a su mundo supernatural. Toda palabra es por sí misma una abstracción. Para decir verdad pueden decir por ejemplo "ua" "ua+gco" "uanai" y otras expresiones al respecto. En cuanto al tiempo, ya puede verse por lo que antecede de este artículo en qué forma tan cabal pueden hacerlo, o si el doctorcito se refiere al tiempo atmosférico también tienen vocablos apropiados para las muchas variaciones del clima. Tienen palabras específicas para los distintos niveles de edad, tales como "jisa" (bebé), "conirue" (joven), "ua+gcuma" (viejo) etc. En cuanto a que no pudieron enamorarse hasta no conocer el español porque su dialecto dizque es carente de dichos vocablos, solo podemos decir que esta sí es la afirmación más ridícula que pueda hacer una persona que ha logrado obtener un título universitario. El amor es un afecto universal que existe hasta en los animales inferiores al hombre, y el Murui-Muinane que es un hombre cabal, íntegro, lo expresa con vocablos tales como "ona isiruittcue" = te quiero mucho, "ona eo gaud+cue" = me gustas mucho "ona o iacad+cue" = me quiero casar contigo. Para no hablar del amor a los niños y las cosas. Respecto a que los sonidos sean guturales y entredientes, vale decir, que esa es la sensación que tenemos todos cuando escuchamos un dialecto nuevo por vez primera. Lo que le pasó al doctorcito fue que no tuvo suficiente motivación para vencer su pereza intelectual y aprender un poquito siquiera. 


\section{BIBLIOGRAFIA}

Fanon, Franz, Escucha Blanco Editorial Nova Terra. Barcelona.

Guzmán Gómez. Ney. Estudio Médico-Antropológico de la Amazonia Colombiana

Universidad del Valle. Facul tad de Medicina. Cali, Colombia. 1968 